\title{
Regression Analysis and Docking Study of Pyrimidine Based Compounds as anti-Tuberculosis Therapeutic Agents
}

\author{
${ }^{1}$ A. K. Parmar, ${ }^{2 *}$ M. R. Patle \\ ${ }^{\mathbf{1 , 2}}$ Department of Chemistry, D. B. Science College, Gondia \\ *Corresponding Author: manojpatle14@gmail.com, Tel: +91-9372736444
}

Available online at: www.isroset.org

Received: 09/Apr/2019, Accepted: 19/Apr/2019, Online: 30/Apr/2019

\begin{abstract}
In the drug-design process, structure activity relationship is an important tool for estimation of biological activity of the unknown compounds. In this process, the objective is development of a relationship between structural features of molecules and the property of interest i. e. biological activity. On the basis of this relationship, the biological activity can be predicted for new candidate structures. Initially, the forty two substituted pyrimidine molecules with known biological activities were considered as known set for regression analysis model building purpose. The properties module from Datawarrior used to calculate descriptors. Structure activity model indicates that these descriptors have significant relationships with observed bioactivity. We have observed a high relationship between experimental and predicted activity values, indicating the validation and the excellent quality of the derived model. In the present study, the new substituted pyrimidine molecules are designed, optimized and their descriptors were calculated using Datawarrior modules. Then by using the Regression analysis model, their biological activities are studied as well as inhibition studies for the 1QPQ by molecular docking method are also carried out. Thus on the basis of regression analysis study and docking study of substituted pyrimidine derivatives, we can conclude that these compounds on further studies may prove to be therapeutic agent against tuberculosis.
\end{abstract}

Keywords: Structure activity, biological activity, docking, tuberculosis, descriptor, pyrimidine..

\section{INTRODUCTION}

In the drug-design process, quantitative structure activity relationship (QSAR) has come to play a major role. In this process, the objective is development of a relationship between structural features and the property of interest, so that property values can be predicted for new candidate structures. [1] The goal of QSAR modeling is to establish a trend in the descriptor values, which parallels the trend in biological activity. [2]

As per the techniques developed in the recent period, the experimental property values have been related directly to structure information. The structure of the molecule is represented in a mathematical manner so that necessary information can be encoded and extracted in a form that lends itself to modeling. In this process, it is expected that the significant structural features are encoded in the structure representation and then identified in the modeling process. In this manner, the synthesis of new candidates may be guided towards the desired goal.

The structure-based approach is a coherent approach to the QSAR problem that has been developed over the past 25 years, and is part of a broader approach, the so-called Quantitative Information Analysis (QIA) [3].

In the QIA approach, emphasis is placed on the two aspects of the data that are known directly, the measured activity and/or property values on the one hand, and the molecular structures in the data set on the other. The required information is related to the manner in which molecules present themselves to each other in non-covalent interactions. It now appears clear that this approach can be accomplished without the need for explicit three-dimensional (3D) structure information. The necessary information is implicit in the encoded descriptors. It should be pointed out that topological structure descriptors are used to produce good predictive models for $\log$ P. [4] [5] [6] [7]

Pyrimidine is an important precursor for the synthesis of a wide variety of heterocyclic compounds. The variety of compounds synthesized reported to have various biological anticancer, antiviral, antibacterial, antioxidant, antituberculosis and antidepressant. [8]

Compounds with heteroatom are exist in nature and have significance as their structure exist in many natural products 
such as vitamins, hormones, and antibiotics and hence they are used in the design of biologically active molecules. Some substituted pyrimidine derivatives were also found to show antibacterial and antifungal activity. [9] [10]

Datawarrior version 4.6.1 package [11] is able to calculate certain physico-chemical properties, lead- or drug-likeness related parameters, ligand efficiencies, various atom and ring counts, molecular shape, flexibility and complexity as well as indications for potential structure activity.

In current study, the experimental work consist initially the equation (model) building for regression analysis by using known set of molecules. By using this equation, the biological activities for newly designed (unknown) molecules are determined. These newly designed molecules are also subjected to inhibition studies against Quinolinic acid phosphoribosyl transferase (QAPRTase) enzyme (PDB code: 1QPQ), an important target for designing novel potential inhibitor for tuberculosis.

\section{EXPERIMENTAL}

The activity parameter used in this study is substituted pyrimidine inhibitory activity. The studied compounds are
Tuberculosis inhibitors which inhibit Mycobacterium Tuberculosis. Interestingly, all these compounds were active and showed M. Tuberculosis inhibition with biological activities values ranged between 374 and $16 \mu \mathrm{M}$. [12] [13]

IIa. Descriptors generation

Firstly, the forty two investigated molecules were preoptimized by means of the Molecular Mechanics. After that, the resulted minimized structures were further refined using the semi-empirical techniques. Then, these substituted pyrimidines were re-optimized by using Gaussian program package.

The QSAR properties module from Datawarrior version 4.6.1 package was used to calculate: Total Molecular Weight, partition coefficient octanol/water (clogP), Aqueous Solubility (cLogS), Polar Surface Area, Fragment-based Drug-Likeness Prediction (LE), Ligand Efficiency (LE), lipophilic ligand Efficiency (LLE), Ligand Efficiency lipophilic price (LELP).

IIb. Regression analysis

Multiple linear regression analysis of molecular descriptors was carried out using the stepwise strategy in SPSS version 19 for Windows.

Table 1.1: Parameters of regression analysis by SPSS

\begin{tabular}{|c|c|c|c|c|c|c|c|c|c|c|c|c|}
\hline \multirow[b]{2}{*}{ Model } & \multicolumn{3}{|c|}{$\begin{array}{l}\text { Unstandardized Coefficients } \\
\text { /Standardized Coefficients }\end{array}$} & \multirow[b]{2}{*}{$\mathrm{t}$} & \multirow[b]{2}{*}{ Sig. } & \multicolumn{2}{|c|}{$\begin{array}{l}\text { 95.0\% Confidence } \\
\text { Interval for B }\end{array}$} & \multicolumn{3}{|c|}{ Correlations } & \multicolumn{2}{|c|}{$\begin{array}{l}\text { Collinearity } \\
\text { Statistics }\end{array}$} \\
\hline & B & $\begin{array}{c}\text { Std. } \\
\text { Error }\end{array}$ & Beta & & & $\begin{array}{l}\text { Lower } \\
\text { Bound }\end{array}$ & $\begin{array}{l}\text { Upper } \\
\text { Bound }\end{array}$ & $\begin{array}{l}\text { Zero } \\
\text { order }\end{array}$ & Partial & Part & Tolerance & VIF \\
\hline $\begin{array}{l}\text { Constan } \\
\mathrm{t}\end{array}$ & -320.28 & 996.680 & & -.321 & .750 & -2345.782 & 1705.21 & & & & & \\
\hline TMW & -.447 & 1.286 & -.210 & -.348 & .730 & -3.061 & 2.167 & -.128 & -.060 & -.054 & .066 & 15.069 \\
\hline cLogP & -92.618 & 72.833 & -1.073 & -1.272 & .212 & -240.633 & 55.397 & -.160 & -.213 & -.198 & .034 & 29.303 \\
\hline $\operatorname{cLog} S$ & -18.068 & 44.693 & -.154 & -.404 & .689 & -108.895 & 72.759 & .135 & -.069 & -.063 & .167 & 5.994 \\
\hline TSA & .566 & 2.872 & .178 & .197 & .845 & -5.271 & 6.403 & -.141 & .034 & .031 & .030 & 33.695 \\
\hline $\begin{array}{l}\text { Drug- } \\
\text { likeness }\end{array}$ & -2.948 & 7.699 & -.089 & -.383 & .704 & -18.595 & 12.699 & -.105 & -.066 & -.060 & .452 & 2.214 \\
\hline LE & 1171.113 & 887.873 & .729 & 1.319 & .196 & -633.263 & 2975.48 & .233 & .221 & .206 & .080 & 12.553 \\
\hline LELP & 30.258 & 28.549 & 1.353 & 1.060 & .297 & -27.761 & 88.277 & -.117 & .179 & .165 & .015 & 67.064 \\
\hline
\end{tabular}

The equation for determination of biological activity generated by regression analysis:

Biological Activity $=(-320.284)+(-.447) \times$ Total Mol. Wt. $+(-92.618) \times \operatorname{cLog} P+(-18.068) \times \operatorname{cLogS}+(.566) \times$ Total Surface area + (-2.948) $x$ Drug likeliness + (1171.11) $\times$ LE $+(30.258) \times$ LELP.

IIc. Docking Studies

Quinolinic acid Phosphoribosyltransferase (QAPRTase) having PDB code $1 \mathrm{QPQ}$ was selected as the target enzyme. Its 3D electronic structure having natural inhibitor was procured from protein repository databank. Quinolinic acid phosphoribosyl transferase (QAPRTase) enzyme (PDB code: 1QPQ) can stop the FAS I pathway as it will make it deficient of NAD.[14] Therefore the Quinolinic acid phosphoribosyl transferase (QAPRTase) enzyme provides an attractive target for designing novel potential inhibitor for tuberculosis. [15].

iGEMDOCK is an integrated tool that creates virtual screening environment from preparations through postscreening analysis with pharmacological interactions. First, iGEMDOCK provides interactive interfaces to prepare both 
the binding site of the target protein and the screening compound library. Then, each compound in the library is docked into the binding site by using the docking tool iGEMDOCK. Subsequently, iGEMDOCK generates proteincompound interaction profiles of electrostatic, hydrogenbonding, and van der Waals interactions. Finally, iGEMDOCK ranks and visualizes the screening compounds by combining the pharmacological interactions and energybased scoring function of iGEMDOCK. [16] The selected set of three ligands were subjected to accurate docking (very slow docking) by setting population size of 700 is set with 70 generation and 10 solutions. After the completion of the docking, the post docking analysis was performed to find the docking pose and its energy values.

\section{RESULTS AND DISCUSSION}

IIIa. Structure activity relationships (SAR)

We have studied eight physical chemical proprieties of series of substituted pyrimidine derivatives in which various degrees of substituents on aromatic ring have been introduced, these substituents include electron donating group such as methoxy and electron withdrawing group like nitro, using HyperChem software. The structure for substituted pyrimidine given as:

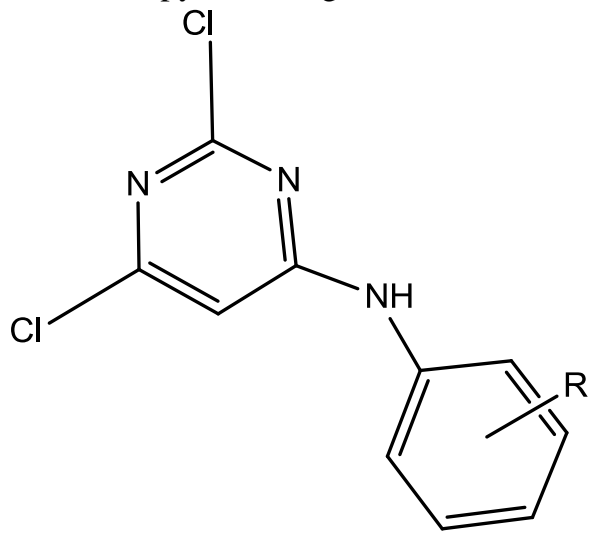

Table 1.2 and 1.3 list the series of substituted pyrimidine derivatives designed in current project.

\begin{tabular}{|c|c|c|c|c|c|}
\hline $\begin{array}{l}\text { Sr } \\
\text { No. }\end{array}$ & ID & Compound & $\begin{array}{l}\text { Sr } \\
\text { No. }\end{array}$ & ID & Compound \\
\hline 1 & UK pyr 1 & N-phenylpyrimidin-4-amine & 29 & UK pyr 29 & $\begin{array}{l}\text { 2-(2-aminoethyl)-N-phenylpyrimidin-4- } \\
\text { amine }\end{array}$ \\
\hline 2 & UK pyr 2 & $\begin{array}{l}\text { 2-methyl-N-phenylpyrimidin-4- } \\
\text { amine }\end{array}$ & 30 & UK pyr 30 & $\begin{array}{l}\text { 6-(2-aminoethyl)-N-phenylpyrimidin-4- } \\
\text { amine }\end{array}$ \\
\hline 3 & UK pyr 3 & $\begin{array}{l}\text { 2,6-dimethyl-N-phenylpyrimidin-4- } \\
\text { amine }\end{array}$ & 31 & UK pyr 31 & $\begin{array}{l}\text { 2,2'-(6-(phenylamino)pyrimidine-2,4- } \\
\text { diyl)diethanamine }\end{array}$ \\
\hline 4 & UK pyr 4 & 6-methyl-N-phenylpyrimidin-4-amine & 32 & UK pyr 32 & $\begin{array}{l}\text { N-(4-(phenylamino)pyrimidin-2- } \\
\text { yl)acetamide }\end{array}$ \\
\hline 5 & UK pyr 5 & 2-ethyl-N-phenylpyrimidin-4-amine & 33 & UK pyr 33 & $\begin{array}{l}\text { N-(6-(phenylamino)pyrimidin-4- } \\
\text { yl)acetamide }\end{array}$ \\
\hline 6 & UK pyr 6 & 6-ethyl-N-phenylpyrimidin-4-amine & 34 & UK pyr 34 & $\begin{array}{l}\text { N,N'-(6-(phenylamino)pyrimidine-2,4- } \\
\text { diyl)diacetamide }\end{array}$ \\
\hline 7 & UK pyr 7 & 2,6-diethyl-N-phenylpyrimidin-4-amine & 35 & UK pyr 35 & $\begin{array}{l}\mathrm{N}-(4-(\text { phenylamino)pyrimidin-2- } \\
\text { yl)propionamide }\end{array}$ \\
\hline 8 & UK pyr 8 & 2-methoxy-N-phenylpyrimidin-4-amine & 36 & UK pyr 36 & $\begin{array}{l}\mathrm{N}-(6-(\text { phenylamino)pyrimidin-4- } \\
\text { yl)propionamide }\end{array}$ \\
\hline 9 & UK pyr 9 & 6-methoxy-N-phenylpyrimidin-4-amine & 37 & UK pyr 37 & $\begin{array}{l}\text { N,N'-(6-(phenylamino)pyrimidine-2,4- } \\
\text { diyl)dipropionamide }\end{array}$ \\
\hline 10 & UK pyr 10 & $\begin{array}{l}\text { 2,6-dimethoxy-N-phenylpyrimidin-4- } \\
\text { amine }\end{array}$ & 38 & UK pyr 38 & $\begin{array}{l}\mathrm{N}-(4-(\text { phenylamino)pyrimidin-2- } \\
\text { yl)butyramide }\end{array}$ \\
\hline 11 & UK pyr 11 & 2-ethoxy-N-phenylpyrimidin-4-amine & 39 & UK pyr 39 & $\begin{array}{l}\mathrm{N}-(6-(\text { phenylamino)pyrimidin-4- } \\
\text { yl)butyramide }\end{array}$ \\
\hline 12 & UK pyr 12 & 6-ethoxy-N-phenylpyrimidin-4-amine & 40 & UK pyr 40 & $\begin{array}{l}\text { N,N'-(6-(phenylamino)pyrimidine-2,4- } \\
\text { diyl)dibutyramide }\end{array}$ \\
\hline 13 & UK pyr 13 & $\begin{array}{l}\text { 2,6-diethoxy-N-phenylpyrimidin-4- } \\
\text { amine }\end{array}$ & 41 & UK pyr 41 & 4-(phenylamino)pyrimidin-2-yl acetate \\
\hline 14 & UK pyr 14 & N-phenyl-2-propylpyrimidin-4-amine & 42 & UK pyr 42 & 6-(phenylamino)pyrimidin-4-yl acetate \\
\hline 15 & UK pyr 15 & N-phenyl-6-propylpyrimidin-4-amine & 43 & UK pyr 43 & 6-(phenylamino)pyrimidine-2,4-diyl \\
\hline
\end{tabular}




\begin{tabular}{|c|l|l|c|l|l|}
\hline & & & & & diacetate \\
\hline 16 & UK pyr 16 & $\begin{array}{l}\text { N-phenyl-2,6-dipropylpyrimidin-4- } \\
\text { amine }\end{array}$ & 44 & UK pyr 44 & $\begin{array}{l}\text { 4-(phenylamino)pyrimidin-2-yl } \\
\text { propionate }\end{array}$ \\
\hline 17 & UK pyr 17 & N-phenyl-2-propoxypyrimidin-4-amine & 45 & UK pyr 45 & $\begin{array}{l}\text { 6-(phenylamino)pyrimidin-4-yl } \\
\text { propionate }\end{array}$ \\
\hline 18 & UK pyr 18 & N-phenyl-6-propoxypyrimidin-4-amine & 46 & UK pyr 46 & $\begin{array}{l}\text { 6-(phenylamino)pyrimidine-2,4-diyl } \\
\text { dipropionate }\end{array}$ \\
\hline 19 & UK pyr 19 & $\begin{array}{l}\text { N-phenyl-2,6-dipropoxypyrimidin-4- } \\
\text { amine }\end{array}$ & 47 & UK pyr 47 & 4-(phenylamino)pyrimidin-2-yl butyrate \\
\hline 20 & UK pyr 20 & 2-butoxy-N-phenylpyrimidin-4-amine & 48 & UK pyr 48 & 6-(phenylamino)pyrimidin-4-yl butyrate \\
\hline 21 & UK pyr 21 & 6-butoxy-N-phenylpyrimidin-4-amine & 49 & UK pyr 49 & $\begin{array}{l}\text { 6-(phenylamino)pyrimidine-2,4-diyl } \\
\text { dibutyrate }\end{array}$ \\
\hline 22 & UK pyr 22 & $\begin{array}{l}\text { 2,6-dibutoxy-N-phenylpyrimidin-4- } \\
\text { amine }\end{array}$ & 50 & UK pyr 50 & 4-(phenylamino)pyrimidin-2-ol \\
\hline 23 & UK pyr 23 & N4-phenylpyrimidine-2,4-diamine & 51 & UK pyr 51 & 6-(phenylamino)pyrimidin-4-ol \\
\hline 24 & UK pyr 24 & N4-phenylpyrimidine-4,6-diamine & 52 & UK pyr 52 & 6-(phenylamino)pyrimidine-2,4-diol \\
\hline 25 & UK pyr 25 & N4-phenylpyrimidine-2,4,6-triamine & 53 & UK pyr 53 & N,2-diphenylpyrimidin-4-amine \\
\hline 26 & UK pyr 26 & $\begin{array}{l}\text { 2-(aminomethyl)-N-phenylpyrimidin-4- } \\
\text { amine }\end{array}$ & 54 & UK pyr 54 & N,6-diphenylpyrimidin-4-amine \\
\hline 27 & UK pyr 27 & $\begin{array}{l}\text { 6-(aminomethyl)-N-phenylpyrimidin-4- } \\
\text { amine }\end{array}$ & 55 & UK pyr 55 & N,2,6-triphenylpyrimidin-4-amine \\
\hline 28 & UK pyr 28 & $\begin{array}{l}\text { 6-(phenylamino)pyrimidine-2,4- } \\
\text { diyl)dimethanamine }\end{array}$ & & & \\
\hline
\end{tabular}

\begin{tabular}{|c|c|c|c|c|c|}
\hline $\begin{array}{l}\text { Sr. } \\
\text { No. }\end{array}$ & Molecule & Molecule name & $\begin{array}{l}\text { Sr. } \\
\text { No. }\end{array}$ & Molecule & Molecule name \\
\hline 1 & UK pyrs1 & $\begin{array}{l}\text { 2,6-dichloro-N-(2- } \\
\text { nitrophenyl)pyrimidin-4-amine }\end{array}$ & 12 & $\begin{array}{c}\mathrm{UK} \\
\text { pyrs12 }\end{array}$ & $\begin{array}{l}\text { 1-(4-((2,6-dichloropyrimidin-4- } \\
\text { yl)amino)phenyl)ethanone }\end{array}$ \\
\hline 2 & UK pyrs2 & $\begin{array}{l}\text { 2,6-dichloro-N-(4- } \\
\text { nitrophenyl)pyrimidin-4-amine }\end{array}$ & 13 & $\begin{array}{c}\text { UK } \\
\text { pyrs13 }\end{array}$ & $\begin{array}{l}\text { 2,6-dichloro-N-(o-tolyl)pyrimidin-4- } \\
\text { amine }\end{array}$ \\
\hline 3 & UK pyrs3 & $\begin{array}{l}\text { 2-((2,6-dichloropyrimidin-4- } \\
\text { yl)amino)benzenesulfonic acid }\end{array}$ & 14 & $\begin{array}{c}\mathrm{UK} \\
\text { pyrs14 }\end{array}$ & $\begin{array}{l}\text { 2,6-dichloro-N-(p-tolyl)pyrimidin-4- } \\
\text { amine }\end{array}$ \\
\hline 4 & UKpyrs4 & $\begin{array}{l}\text { 4-((2,6-dichloropyrimidin-4- } \\
\text { yl)amino)benzenesulfonic acid }\end{array}$ & 15 & $\begin{array}{l}\text { UK } \\
\text { pyrs15 }\end{array}$ & $\begin{array}{l}\text { 2-((2,6-dichloropyrimidin-4- } \\
\text { yl)amino)phenol }\end{array}$ \\
\hline 5 & UKpyrs5 & $\begin{array}{l}\text { 2,6-dichloro-N-(2- } \\
\text { chlorophenyl)pyrimidin-4-amine }\end{array}$ & 16 & $\begin{array}{c}\text { UK } \\
\text { pyrs16 }\end{array}$ & $\begin{array}{l}\text { 4-((2,6-dichloropyrimidin-4- } \\
\text { yl)amino)phenol }\end{array}$ \\
\hline 6 & UK pyrs6 & $\begin{array}{l}\text { 2,6-dichloro-N-(4- } \\
\text { chlorophenyl)pyrimidin-4-amine }\end{array}$ & 17 & $\begin{array}{c}\text { UK } \\
\text { pyrs17 }\end{array}$ & $\begin{array}{l}\text { N1-(2,6-dichloropyrimidin-4-yl)benzene- } \\
\text { 1,2-diamine }\end{array}$ \\
\hline 7 & UK pyrs7 & $\begin{array}{l}\text { N-(2-bromophenyl)-2,6- } \\
\text { dichloropyrimidin-4-amine }\end{array}$ & 18 & $\begin{array}{c}\text { UK } \\
\text { pyrs } 18\end{array}$ & $\begin{array}{l}\text { N1-(2,6-dichloropyrimidin-4-yl)benzene- } \\
\text { 1,4-diamine }\end{array}$ \\
\hline 8 & UK pyrs8 & $\begin{array}{l}\text { N-(4-bromophenyl)-2,6- } \\
\text { dichloropyrimidin-4-amine }\end{array}$ & 19 & $\begin{array}{c}\text { UK } \\
\text { pyrs19 }\end{array}$ & $\begin{array}{l}\text { 2,6-dichloro-N-(2,4,6- } \\
\text { trichlorophenyl)pyrimidin-4-amine }\end{array}$ \\
\hline 9 & UK pyrs9 & $\begin{array}{l}\text { 2,6-dichloro-N-(2- } \\
\text { iodophenyl)pyrimidin-4-amine }\end{array}$ & 20 & $\begin{array}{c}\text { UK } \\
\text { pyrs20 }\end{array}$ & $\begin{array}{l}\text { 2,6-dichloro-N-(2,4,6- } \\
\text { triiodophenyl)pyrimidin-4-amine }\end{array}$ \\
\hline 10 & UK pyrs10 & $\begin{array}{l}\text { 2,6-dichloro-N-(4- } \\
\text { iodophenyl)pyrimidin-4-amine }\end{array}$ & 21 & $\begin{array}{c}\mathrm{UK} \\
\text { pyrs21 }\end{array}$ & $\begin{array}{l}\text { 2,6-dichloro-N-(naphthalen-1- } \\
\text { yl)pyrimidin-4-amine }\end{array}$ \\
\hline 11 & UK pyrs11 & $\begin{array}{l}\text { 1-(2-((2,6-dichloropyrimidin-4- } \\
\text { yl)amino)phenyl)ethanone }\end{array}$ & & & \\
\hline
\end{tabular}

Table 1.4 shows the observed biological activity values of known molecules. 
Table 1.4: Descriptor values with biological activity for Known set of molecules.

\begin{tabular}{|c|c|c|c|c|c|c|c|c|c|}
\hline ID & $\begin{array}{c}\text { Observed } \\
\text { Biological } \\
\text { Activity } \\
\end{array}$ & Total Mol. Wt. & cLogP & cLogS & $\begin{array}{c}\text { Total } \\
\text { Surface } \\
\text { Area }\end{array}$ & Drug likeness & $\begin{array}{c}\text { LE from } \\
\text { Total Mol. } \\
\text { Wt. }\end{array}$ & $\begin{array}{c}\text { LLE from } \\
\text { Total Mol. } \\
\text { Wt. }\end{array}$ & $\begin{array}{c}\text { LELP from } \\
\text { Total Mol. } \\
\text { Wt. }\end{array}$ \\
\hline K PYR 1 & 331 & 219.674 & 3.2242 & -3.588 & 169.68 & -0.90842 & 0.60895 & 3.434 & 5.2947 \\
\hline K PYR 2 & 165.5 & 256.352 & 3.4546 & -3.99 & 216.6 & 1.9941 & 0.47591 & 3.1366 & 7.2589 \\
\hline K PYR 3 & 331 & 270.379 & 2.4796 & -4.468 & 227.7 & 2.7554 & 0.45053 & 4.0884 & 5.5038 \\
\hline K PYR 4 & 82.75 & 296.417 & 4.2271 & -4.776 & 244.62 & 4.4193 & 0.40708 & 2.301 & 10.384 \\
\hline K PYR 5 & 82.75 & 310.443 & 3.2521 & -5.254 & 255.72 & 5.0045 & 0.38818 & 3.2559 & 8.3777 \\
\hline K PYR 6 & 20.65 & 352.524 & 4.2838 & -6.286 & 292.5 & 5.0045 & 0.34048 & 2.169 & 12.582 \\
\hline K PYR 7 & 374.5 & 220.299 & 2.6847 & -4.099 & 162.84 & -6.8696 & 0.60884 & 3.9723 & 4.4095 \\
\hline K PYR 8 & 75.9 & 308.408 & 5.9035 & -5.838 & 238.84 & -1.3412 & 0.40601 & 0.60738 & 14.54 \\
\hline K PYR 9 & 250 & 322.435 & 6.2474 & -6.182 & 251.1 & -1.4387 & 0.3872 & 0.24416 & 16.135 \\
\hline K PYR 10 & 250 & 336.462 & 6.663 & -6.341 & 264.86 & -1.6789 & 0.37001 & -0.18993 & 18.008 \\
\hline K PYR 11 & 72.1 & 324.407 & 5.5578 & -5.542 & 245.19 & -1.4453 & 0.38704 & 0.93111 & 14.36 \\
\hline K PYR 12 & 71.2 & 338.434 & 5.8335 & -5.856 & 261.1 & -1.4875 & 0.36987 & 0.63703 & 15.772 \\
\hline K PYR 13 & 119.4 & 338.434 & 5.8335 & -5.856 & 261.1 & -1.4875 & 0.36987 & 0.63703 & 15.772 \\
\hline K PYR 14 & 250 & 352.417 & 6.0149 & -6.549 & 259.1 & -1.5895 & 0.35411 & 0.43804 & 16.986 \\
\hline K PYR 15 & 250 & 368.46 & 5.7635 & -5.874 & 283.36 & -1.4875 & 0.33947 & 0.67011 & 16.978 \\
\hline K PYR 16 & 250 & 398.486 & 5.6935 & -5.892 & 305.62 & -1.4875 & 0.31355 & 0.70609 & 18.158 \\
\hline K PYR 17 & 250 & 354.433 & 5.4878 & -5.56 & 267.45 & -1.4988 & 0.35397 & 0.96267 & 15.504 \\
\hline K PYR 18 & 250 & 384.459 & 5.4178 & -5.578 & 289.71 & -1.4988 & 0.32596 & 0.99735 & 16.621 \\
\hline K PYR 19 & 200 & 499.432 & 7.8391 & -7.726 & 354.07 & -8.9071 & 0.27887 & -1.5376 & 28.11 \\
\hline K PYR 20 & 250 & 353.405 & 4.9819 & -6.298 & 262.51 & -6.5934 & 0.35404 & 1.4698 & 14.072 \\
\hline K PYR 21 & 250 & 353.405 & 4.9819 & -6.298 & 262.51 & -6.5934 & 0.35404 & 1.4698 & 14.072 \\
\hline K PYR 22 & 16.2 & 376.405 & 6.7518 & -6.616 & 268.3 & -8.5312 & 0.33898 & -0.32745 & 19.918 \\
\hline K PYR 23 & 15.7 & 376.405 & 6.7518 & -6.616 & 268.3 & -8.5312 & 0.33898 & -0.32745 & 19.918 \\
\hline K PYR 24 & 71.1 & 326.398 & 6.0043 & -6.152 & 245.19 & -2.6812 & 0.38689 & 0.48195 & 15.52 \\
\hline K PYR 25 & 300 & 326.398 & 6.0043 & -6.152 & 245.19 & -2.6812 & 0.38689 & 0.48195 & 15.52 \\
\hline K PYR 26 & 250 & 342.853 & 6.5095 & -6.574 & 254.26 & -1.3257 & 0.38561 & -0.04461 & 16.881 \\
\hline K PYR 27 & 124.3 & 342.853 & 6.5095 & -6.574 & 254.26 & -1.3257 & 0.38561 & -0.04461 & 16.881 \\
\hline K PYR 28 & 250 & 387.304 & 6.6287 & -6.672 & 257.47 & -3.1312 & 0.38245 & -0.21675 & 17.332 \\
\hline K PYR 29 & 24.3 & 387.304 & 6.6287 & -6.672 & 257.47 & -3.1312 & 0.38245 & -0.21675 & 17.332 \\
\hline K PYR 30 & 300 & 333.418 & 5.7391 & -6.611 & 260.55 & -5.6212 & 0.37024 & 0.73791 & 15.501 \\
\hline K PYR 31 & 250 & 372.495 & 6.7851 & -7.331 & 287.2 & -1.3412 & 0.32665 & -0.35622 & 20.772 \\
\hline K PYR 32 & 250 & 372.495 & 6.7851 & -7.331 & 287.2 & -1.3412 & 0.32665 & -0.35622 & 20.772 \\
\hline K PYR 33 & 35 & 362.5 & 6.8306 & -6.67 & 292.86 & -5.6302 & 0.33984 & -0.38991 & 20.099 \\
\hline K PYR 34 & 39.9 & 312.396 & 4.7794 & -5.407 & 242.29 & -1.67 & 0.40566 & 1.7259 & 11.782 \\
\hline K PYR 35 & 20.8 & 311.412 & 4.3672 & -4.773 & 242.17 & -0.47093 & 0.40574 & 2.1395 & 10.763 \\
\hline K PYR 36 & 300 & 400.509 & 5.1165 & -5.284 & 308.62 & -1.3412 & 0.30264 & 1.2809 & 16.906 \\
\hline K PYR 37 & 145.1 & 323.423 & 4.5898 & -4.93 & 251.36 & -1.3412 & 0.38712 & 1.9004 & 11.856 \\
\hline K PYR 38 & 299.2 & 302.445 & 5.8417 & -5.467 & 240.03 & -4.8911 & 0.42589 & 0.67765 & 13.716 \\
\hline K PYR 39 & 239.7 & 274.391 & 4.8215 & -5.02 & 216.1 & -1.7595 & 0.47378 & 1.7401 & 10.177 \\
\hline K PYR 40 & 72.6 & 336.462 & 6.0182 & -6.093 & 263.6 & -1.3447 & 0.37001 & 0.45487 & 16.265 \\
\hline K PYR 41 & 160.2 & 302.445 & 5.7303 & -5.56 & 243.62 & -1.4275 & 0.42589 & 0.78905 & 13.455 \\
\hline K PYR 42 & 250 & 398.533 & 7.1183 & -6.874 & 311.1 & -1.3412 & 0.30274 & -0.71876 & 23.513 \\
\hline
\end{tabular}

Table 1.5 and 1.6 shows the calculated biological activity values by SPSS of unknown molecules Series I and Series II. 
Table 1.5: Calculated biological activity values by SPSS of unknown molecules Series I

\begin{tabular}{|c|c|c|c|c|c|c|c|c|c|}
\hline ID & $\begin{array}{c}\text { Calculated } \\
\text { Biological } \\
\text { Activity }\end{array}$ & $\begin{array}{c}\text { Total Mol. } \\
\text { Wt. }\end{array}$ & cLogP & cLogS & $\begin{array}{c}\text { Total } \\
\text { Surface } \\
\text { Area }\end{array}$ & $\begin{array}{c}\text { Drug } \\
\text { likeness }\end{array}$ & $\begin{array}{l}\text { LE from } \\
\text { Total } \\
\text { Mol. Wt. }\end{array}$ & $\begin{array}{c}\text { LLE from } \\
\text { Total Mol. } \\
\text { Wt. }\end{array}$ & $\begin{array}{c}\text { LELP from } \\
\text { Total Mol. } \\
\text { Wt. }\end{array}$ \\
\hline UK pyr 2 & 563.568 & 185.229 & 2.2116 & -2.343 & 154.26 & -1.0024 & 0.65971 & 4.5207 & 3.3524 \\
\hline UK pyr 3 & 519.0692 & 199.256 & 2.6095 & -2.711 & 166.52 & -1.0024 & 0.61283 & 4.0911 & 4.2581 \\
\hline UK pyr 5 & 516.3739 & 199.256 & 2.6272 & -2.502 & 168.02 & -1.3406 & 0.61283 & 4.0734 & 4.287 \\
\hline UK pyr 6 & 522.6277 & 199.256 & 2.7917 & -3.242 & 168.02 & -1.3406 & 0.61283 & 3.9089 & 4.5554 \\
\hline UK pyr 7 & 452.4304 & 227.31 & 3.4407 & -3.029 & 194.04 & -1.3406 & 0.53611 & 3.2027 & 6.4179 \\
\hline UK pyr 8 & 537.7487 & 201.228 & 2.2777 & -3.004 & 164.26 & -0.93934 & 0.61244 & 4.4186 & 3.7191 \\
\hline UK pyr 12 & 511.6471 & 215.255 & 2.6659 & -3.558 & 178.02 & -2.6308 & 0.57165 & 4.0011 & 4.6635 \\
\hline UK pyr 13 & 457.8591 & 259.308 & 3.3717 & -4.147 & 214.04 & -2.6308 & 0.47555 & 3.2145 & 7.0901 \\
\hline UK pyr 14 & 484.8035 & 213.283 & 3.0816 & -2.772 & 181.78 & -3.4155 & 0.57199 & 3.5894 & 5.3875 \\
\hline UK pyr 15 & 491.6381 & 213.283 & 3.2461 & -3.512 & 181.78 & -3.4155 & 0.57199 & 3.4249 & 5.6751 \\
\hline UK pyr 16 & 424.1414 & 255.364 & 4.3495 & -3.569 & 221.56 & -3.4155 & 0.47603 & 2.2433 & 9.137 \\
\hline UK pyr 17 & 473.1969 & 229.282 & 3.1384 & -3.574 & 191.78 & -1.5537 & 0.53581 & 3.5012 & 5.8573 \\
\hline UK pyr 18 & 478.4401 & 229.282 & 3.1203 & -3.828 & 191.78 & -1.5537 & 0.53581 & 3.5193 & 5.8235 \\
\hline UK pyr 19 & 440.4466 & 287.362 & 4.2805 & -4.687 & 241.56 & -1.5537 & 0.42735 & 2.2611 & 10.016 \\
\hline UK pyr 25 & 587.0271 & 201.232 & 1.3445 & -3.663 & 159.04 & -0.9353 & 0.61244 & 5.3518 & 2.1953 \\
\hline UK pyr 26 & 580.063 & 200.244 & 0.8726 & -1.959 & 164.28 & -1.1705 & 0.61263 & 5.8258 & 1.4243 \\
\hline UK pyr 27 & 586.3228 & 200.244 & 1.0371 & -2.699 & 164.28 & -1.1705 & 0.61263 & 5.6613 & 1.6929 \\
\hline UK pyr 28 & 555.6118 & 229.286 & 0.0685 & -1.943 & 186.56 & -1.1705 & 0.53581 & 6.7081 & -0.12784 \\
\hline UK pyr 29 & 535.213 & 214.271 & 1.3027 & -2.071 & 178.04 & -1.4642 & 0.57182 & 5.3663 & 2.2782 \\
\hline UK pyr 30 & 542.0475 & 214.271 & 1.4672 & -2.811 & 178.04 & -1.4642 & 0.57182 & 5.2018 & 2.5658 \\
\hline UK pyr 31 & 492.8843 & 257.34 & 0.7917 & -2.167 & 214.08 & -1.4642 & 0.47579 & 5.7978 & 1.664 \\
\hline UK pyr 32 & 497.1944 & 228.254 & 2.0514 & -3.328 & 183.47 & 0.43291 & 0.53597 & 4.5902 & 3.8275 \\
\hline UK pyr 33 & 502.4376 & 228.254 & 2.0333 & -3.582 & 183.47 & 0.43291 & 0.53597 & 4.6083 & 3.7937 \\
\hline UK pyr 34 & 463.0151 & 285.306 & 2.1065 & -4.195 & 224.94 & 0.43291 & 0.42755 & 4.4382 & 4.9269 \\
\hline UK pyr 35 & 467.5092 & 242.281 & 2.5058 & -3.598 & 197.23 & 1.7575 & 0.50422 & 4.1099 & 4.9697 \\
\hline UK pyr 36 & 472.6889 & 242.281 & 2.4877 & -3.852 & 197.23 & 1.7575 & 0.50422 & 4.128 & 4.9338 \\
\hline UK pyr 37 & 452.5068 & 313.36 & 3.0153 & -4.735 & 252.46 & 1.7575 & 0.38794 & 3.4887 & 7.7726 \\
\hline
\end{tabular}




\begin{tabular}{|l|r|r|r|r|r|r|r|r|r|}
\hline UK pyr 38 & 458.4033 & 256.308 & 2.9602 & -3.868 & 210.99 & -1.4189 & 0.47592 & 3.631 & 6.22 \\
\hline UK pyr 39 & 463.5194 & 256.308 & 2.9421 & -4.122 & 210.99 & -1.4189 & 0.47592 & 3.6491 & 6.182 \\
\hline UK pyr 40 & 476.2157 & 341.414 & 3.9241 & -5.275 & 279.98 & -1.4189 & 0.35486 & 2.5426 & 11.058 \\
\hline UK pyr 41 & 490.3734 & 229.238 & 2.3344 & -3.286 & 182.01 & -1.1623 & 0.53582 & 4.3053 & 4.3567 \\
\hline UK pyr 42 & 495.6166 & 229.238 & 2.3163 & -3.54 & 182.01 & -1.1623 & 0.53582 & 4.3234 & 4.3229 \\
\hline UK pyr 43 & 452.9037 & 287.274 & 2.6725 & -4.111 & 222.02 & -1.1623 & 0.42735 & 3.8692 & 6.2536 \\
\hline UK pyr 44 & 459.0787 & 243.265 & 2.7888 & -3.556 & 195.77 & 1.056 & 0.50408 & 3.8251 & 5.5324 \\
\hline UK pyr 45 & 464.2583 & 243.265 & 2.7707 & -3.81 & 195.77 & 1.056 & 0.50408 & 3.8432 & 5.4965 \\
\hline UK pyr 46 & 443.9246 & 315.328 & 3.5813 & -4.651 & 249.54 & 1.056 & 0.38778 & 2.9199 & 9.2354 \\
\hline UK pyr 47 & 457.3393 & 257.292 & 3.2432 & -3.826 & 209.53 & -4.2655 & 0.4758 & 3.3464 & 6.8164 \\
\hline UK pyr 48 & 462.4525 & 257.292 & 3.2251 & -4.08 & 209.53 & -4.2655 & 0.4758 & 3.3645 & 6.7783 \\
\hline UK pyr 49 & 478.1427 & 343.382 & 4.4901 & -5.191 & 277.06 & -4.2655 & 0.35473 & 1.9741 & 12.658 \\
\hline UK pyr 50 & 576.6043 & 187.201 & 2.002 & -2.69 & 148.35 & -0.96557 & 0.65926 & 4.7257 & 3.0368 \\
\hline UK pyr 51 & 582.0381 & 187.201 & 1.9839 & -2.944 & 148.35 & -0.96557 & 0.65926 & 4.7438 & 3.0093 \\
\hline UK pyr 52 & 543.0372 & 203.2 & 2.0077 & -2.919 & 154.7 & -0.96557 & 0.61205 & 4.6844 & 3.2803 \\
\hline UK pyr 53 & 446.8978 & 247.3 & 3.6212 & -4.86 & 201.76 & -0.82797 & 0.47704 & 2.9856 & 7.591 \\
\hline UK pyr 54 & 437.1354 & 247.3 & 3.7284 & -4.493 & 201.76 & -0.82797 & 0.47704 & 2.8784 & 7.8157 \\
\hline UK pyr 55 & 469.7895 & 323.398 & 5.3714 & -6.638 & 261.52 & -0.82797 & 0.35615 & 1.1189 & 15.082 \\
\hline
\end{tabular}

\begin{tabular}{|c|c|c|c|c|c|c|c|c|c|}
\hline \multicolumn{1}{|c|}{ Table 1.6: Calculated biological activity values by SPSS of unknown molecules Series II } \\
\hline ID & $\begin{array}{c}\text { Calculated } \\
\text { Biological } \\
\text { Activity }\end{array}$ & $\begin{array}{c}\text { Total } \\
\text { Molweight }\end{array}$ & cLogP & cLogS & $\begin{array}{c}\text { Total } \\
\text { Surface } \\
\text { Area }\end{array}$ & $\begin{array}{c}\text { Drug } \\
\text { likeness }\end{array}$ & $\begin{array}{c}\text { LE from } \\
\text { Total } \\
\text { Molweight }\end{array}$ & $\begin{array}{c}\text { LLE from } \\
\text { Total } \\
\text { Molweight }\end{array}$ & $\begin{array}{c}\text { LELP from } \\
\text { Total } \\
\text { Molweight }\end{array}$ \\
\hline UK pyrs1 & 206.215 & 285.09 & 1.2693 & -2.05 & 105.95 & -4.7645 & 0.49883 & 5.2757 & 2.5445 \\
\hline UK pyrs2 & 194.8372 & 285.09 & 1.2693 & -2.05 & 105.95 & -0.905 & 0.49883 & 5.2757 & 2.5445 \\
\hline UK pyrs3 & 222.9136 & 320.156 & -1.03 & -1.895 & 115.5 & -3.1459 & 0.46894 & 7.5247 & -2.1967 \\
\hline UK pyrs4 & 211.3626 & 320.156 & -1.03 & -1.895 & 115.5 & 0.77236 & 0.46894 & 7.5247 & -2.1967 \\
\hline UK pyrs5 & 210.1046 & 274.538 & 3.4123 & -3.256 & 98.37 & -4.4182 & 0.56259 & 3.1491 & 6.0653 \\
\hline UK pyrs6 & 198.7395 & 274.538 & 3.4123 & -3.256 & 98.37 & -0.563 & 0.56259 & 3.1491 & 6.0653 \\
\hline UK pyrs7 & 209.1565 & 318.989 & 3.5433 & -3.398 & 101.58 & -12.648 & 0.557 & 2.9529 & 6.3614 \\
\hline UK pyrs8 & 197.7923 & 318.989 & 3.5433 & -3.398 & 101.58 & -8.7931 & 0.557 & 2.9529 & 6.3614 \\
\hline UK pyrs9 & 145.011 & 365.985 & 3.7957 & -2.979 & 109.23 & -3.78 & 0.55188 & 2.6408 & 6.8777 \\
\hline UK pyrs10 & 133.5541 & 365.985 & 3.7957 & -2.979 & 109.23 & 0.10633 & 0.55188 & 2.6408 & 6.8777 \\
\hline UK pyrs11 & 175.3621 & 282.129 & 2.8292 & -2.96 & 105.22 & -5.2336 & 0.49918 & 3.7204 & 5.6677 \\
\hline UK pyrs12 & 163.8772 & 282.129 & 2.8292 & -2.96 & 105.22 & -1.3378 & 0.49918 & 3.7204 & 5.6677 \\
\hline UK pyrs13 & 216.2544 & 254.119 & 3.3966 & -3.089 & 95.21 & -4.0024 & 0.56547 & 3.1984 & 6.0067 \\
\hline UK pyrs14 & 205.7908 & 254.119 & 3.3966 & -3.089 & 95.21 & -0.453 & 0.56547 & 3.1984 & 6.0067 \\
\hline UK pyrs15 & 244.8595 & 256.092 & 2.2752 & -2.53 & 89.3 & -3.7779 & 0.56518 & 4.3164 & 4.0256 \\
\hline UK pyrs16 & 233.1713 & 256.092 & 2.2752 & -2.53 & 89.3 & 0.1869 & 0.56518 & 4.3164 & 4.0256 \\
\hline UK pyrs17 & 277.0168 & 255.108 & 1.8774 & -2.606 & 91.47 & -8.3321 & 0.56532 & 4.7159 & 3.3209 \\
\hline
\end{tabular}




\begin{tabular}{|c|r|c|c|c|c|c|c|c|c|}
\hline UK pyrs18 & 259.6003 & 255.108 & 1.8774 & -2.606 & 91.47 & -2.4242 & 0.56532 & 4.7159 & 3.3209 \\
\hline UK pyrs19 & 117.0722 & 343.428 & 3.9823 & -3.91 & 125.03 & 0.84182 & 0.49267 & 2.4819 & 8.0831 \\
\hline UK pyrs20 & -49.72 & 617.769 & 5.1325 & -3.079 & 157.61 & 1.48 & 0.47324 & 1.0767 & 10.846 \\
\hline UK pyrs21 & 120.5357 & 290.152 & 4.0567 & -3.875 & 90.36 & -4.4182 & 0.47203 & 2.4807 & 8.5942 \\
\hline
\end{tabular}

Table 1.7 and 1.8 shows docking parameters with protein 1QPQ for unknown pyrimidine molecules (Series I and II).

Table 1.7: Docking parameters with protein 1QPQ for unknown pyrimidine molecules (Series I).

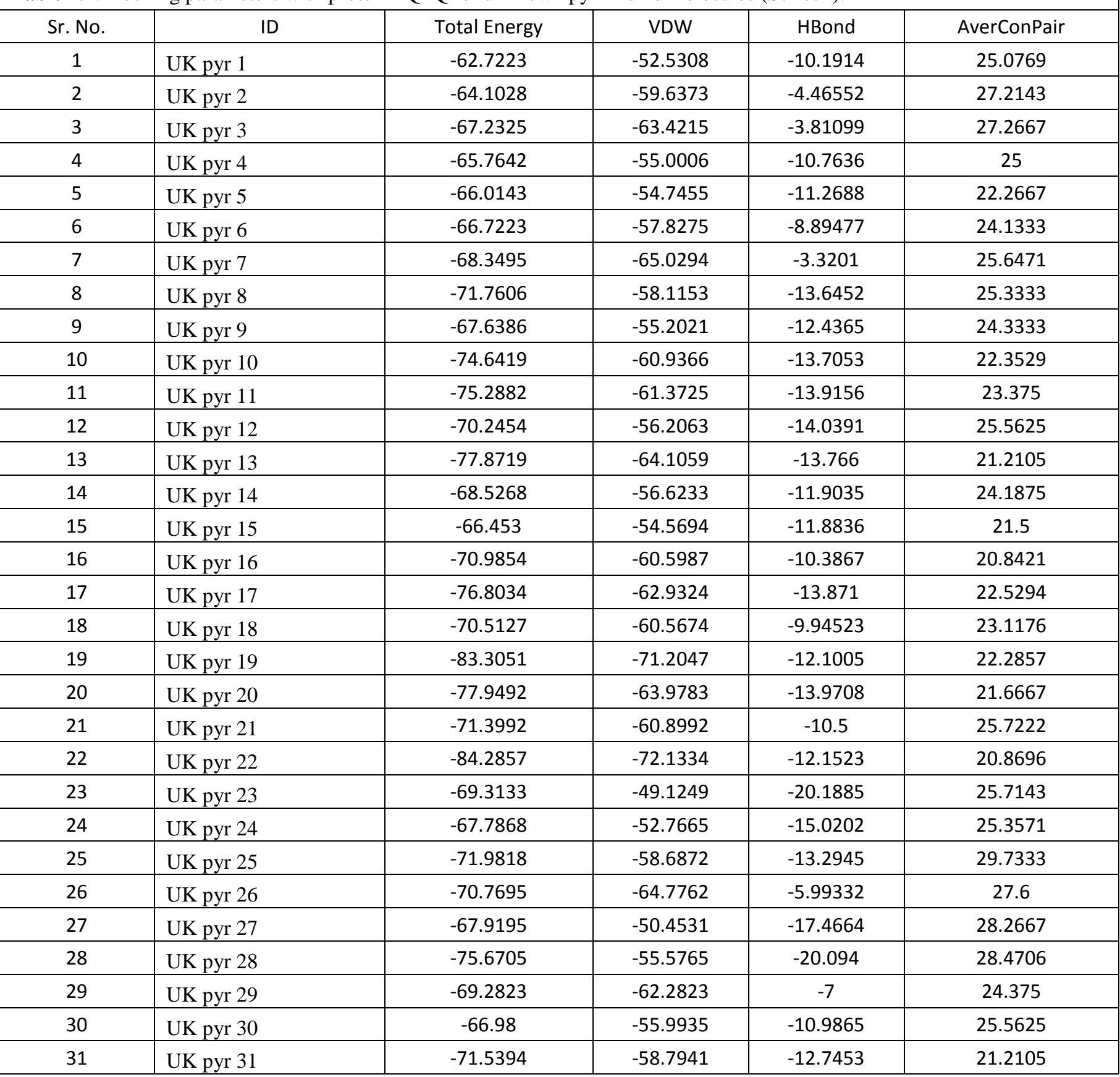




\begin{tabular}{|c|l|c|c|c|c|}
\hline 32 & UK pyr 32 & -81.9606 & -66.3836 & -15.5769 & 26.2941 \\
\hline 33 & UK pyr 33 & -81.1744 & -63.7252 & -17.4491 & 25.3529 \\
\hline 34 & UK pyr 34 & -86.5691 & -66.6472 & -19.9219 & 24.0952 \\
\hline 35 & UK pyr 35 & -85.2654 & -69.7298 & -15.5356 & 25.9444 \\
\hline 36 & UK pyr 36 & -84.4913 & -66.8354 & -17.6559 & 24.2222 \\
\hline 37 & UK pyr 37 & -91.1798 & -71.1819 & -19.9978 & 23.4348 \\
\hline 38 & UK pyr 38 & -82.2284 & -67.5288 & -14.6997 & 25.1053 \\
\hline 39 & UK pyr 39 & -86.0416 & -69.066 & -16.9756 & 23.4737 \\
\hline 40 & UK pyr 40 & -86.5393 & -70.8854 & -15.654 & 18.2 \\
\hline 41 & UK pyr 41 & -78.0141 & -69.1096 & -8.90456 & 26.1765 \\
\hline 42 & UK pyr 42 & -80.7482 & -59.8732 & -20.875 & 23.8824 \\
\hline 43 & UK pyr 43 & -80.5343 & -62.904 & -17.6303 & 19.7619 \\
\hline 44 & UK pyr 44 & -85.3536 & -65.1234 & -20.2302 & 23.2222 \\
\hline 45 & UK pyr 45 & -86.3866 & -65.3825 & -21.0041 & 24.3333 \\
\hline 46 & UK pyr 46 & -96.8059 & -76.4032 & -20.4028 & 23.5217 \\
\hline 47 & UK pyr 47 & -83.5746 & -62.6332 & -20.9414 & 22 \\
\hline 48 & UK pyr 48 & -86.9215 & -67.6668 & -19.2548 & 22.8947 \\
\hline 49 & UK pyr 49 & -91.2584 & -68.5932 & -22.6652 & 18.8 \\
\hline 50 & UK pyr 50 & -69.116 & -55.3228 & -13.7932 & 25.5714 \\
\hline 51 & UK pyr 51 & -68.5876 & -58.0876 & -10.5 & 26.0714 \\
\hline 52 & UK pyr 52 & -74.7601 & -59.6527 & -15.1074 & 25.8667 \\
\hline 53 & UK pyr 53 & -73.3889 & -64.4239 & -8.96498 & 21.2105 \\
\hline 54 & UK pyr 54 & -74.7236 & -64.9488 & -9.77476 & 27.9474 \\
\hline 55 & UK pyr 55 & & & & 17.76 \\
\hline & & & -60.8764 & -10.4745 & \\
\hline
\end{tabular}

Table 1.8: Docking parameters with protein $1 \mathrm{QPQ}$ for unknown pyrimidine molecules (Series II).

\begin{tabular}{|c|c|c|c|c|c|}
\hline Sr. No. & ID & Binding Energy & VDW & HBond & AverConPair \\
\hline 1 & UK pyrs1 & 17.377 & 17.0682 & 0 & 20.5556 \\
\hline 2 & UK pyrs2 & -78.989 & -57.3475 & -23.7755 & 24.5 \\
\hline 3 & UK pyrs3 & -82.1703 & -58.4182 & -15.492 & 25.7368 \\
\hline 4 & UK pyrs4 & -97.84 & -62.8341 & -29.1804 & 24.7895 \\
\hline 5 & UK pyrs5 & -67.0732 & -60.0732 & -7 & 23.5 \\
\hline 6 & UK pyrs6 & -65.5205 & -58.5205 & -7 & 22.8125 \\
\hline 7 & UK pyrs7 & -66.9514 & -59.9514 & -7 & 23.5 \\
\hline 8 & UK pyrs8 & -65.3454 & -57.2472 & -8.09817 & 22.9375 \\
\hline 9 & UK pyrs9 & -66.7527 & -59.0042 & -7.74843 & 23.75 \\
\hline 10 & UK pyrs10 & -65.2178 & -56.4706 & -8.74717 & 22.75 \\
\hline 11 & UK pyrs11 & -43.452 & -37.9882 & -5.46376 & 16.3889 \\
\hline 12 & UK pyrs12 & -82.6416 & -63.853 & -18.7886 & 24.6111 \\
\hline 13 & UK pyrs13 & -67.1661 & -60.1661 & -7 & 23.625 \\
\hline 14 & UK pyrs14 & -66.0166 & -59.0384 & -6.97812 & 27.3125 \\
\hline
\end{tabular}




\begin{tabular}{|l|l|l|l|l|c|}
\hline 15 & UK pyrs15 & -67.1567 & -57.6567 & -9.5 & 21.125 \\
\hline 16 & UK pyrs16 & -67.5031 & -47.797 & -19.7061 & 21.875 \\
\hline 17 & UK pyrs17 & -68.83 & -58.33 & -10.5 & 21.125 \\
\hline 18 & UK pyrs18 & -67.1823 & -60.2069 & -6.97541 & 28 \\
\hline 19 & UK pyrs19 & -69.289 & -62.3609 & -6.92813 & 23.2778 \\
\hline 20 & UK pyrs20 & -66.7187 & -59.0242 & -7.69455 & 23.1667 \\
\hline 21 & UK pyrs21 & -78.1383 & -71.1502 & -6.98812 & 23 \\
\hline
\end{tabular}

Table 1.9 shows summary statistics for the QSAR Model.

Table 1.9: Summary statistics for the QSAR Model.

\begin{tabular}{|c|c|c|c|c|c|c|c|c|c|c|}
\hline \multicolumn{11}{|c|}{ Model Summary } \\
\hline \multirow[b]{2}{*}{ Model } & \multirow[b]{2}{*}{$\mathrm{R}$} & \multirow[b]{2}{*}{ R Square } & \multirow[b]{2}{*}{$\begin{array}{l}\text { Adjusted R } \\
\text { Square }\end{array}$} & \multirow[b]{2}{*}{$\begin{array}{l}\text { Std. Error of } \\
\text { the Estimate }\end{array}$} & \multicolumn{5}{|c|}{ Change Statistics } & \multirow[b]{2}{*}{$\begin{array}{l}\text { Durbin- } \\
\text { Watson }\end{array}$} \\
\hline & & & & & $\begin{array}{l}\text { R Square } \\
\text { Change }\end{array}$ & F Change & df1 & df2 & $\begin{array}{c}\text { Sig. F } \\
\text { Change }\end{array}$ & \\
\hline 1 & $0.416^{\mathrm{a}}$ & 0.173 & 0.003 & 106.687082 & 0.173 & 1.017 & 7 & 34 & 0.437 & 1.746 \\
\hline
\end{tabular}

The values of fraction variance may vary between 0 and 1 . QSAR model having $\mathrm{r}^{2}>0.173$ will only be considered for validation. For example, the value $r=0.416$ and $r^{2}=0.173$ allowed us to indicate firmly the correlation between different parameters (independent variables) with biological activity of the compounds. In equation of biological activity, the negative coefficients of molecular volume (MV) and molecular weight (MW) explain that any increase in molecular volume or molecular weight of the compounds causes a decrease in the biological activity.

\section{CONCLUSIONS}

Based on the present investigation it can be concluded that the equation

"Biological Activity $=(-320.284)+(-.447) \times$ Total Mol. Wt. + (-92.618) $\times$ cLogP + (-18.068) $\times$ cLogS + (.566) $x$ Total Surface area $+(-2.948)$ x Drug likeliness + (1171.11) x LE + (30.258) x LELP."

can be useful for predicting the activity of new substituted pyrimidine derivatives prior to their synthesis.

Structure activity model indicates that these descriptors have significant relationships with observed bioactivity. We have observed a high relationship between experimental and predicted activity values, indicating the validation and the excellent quality of the derived model.

In equation of biological activity, the negative coefficients of surface area that any increase in surface area of the molecules causes a decrease in the biological activity.

As well as, the inhibition of Quinolinic acid Phosphoribosyltransferase (QAPRTase) having PDB code $1 Q P Q$ proteins can be an effective drug in the prevention and treatment of tuberculosis. In the present study, the ligands were generated and were studied for its ability to inhibit the 1QPQ by molecular docking method. The ligands with good inhibitory properties were generated among which UK pyr 46 , UK pyr 49 , UK pyr 37 , UK pyrs 3 and UK pyrs4 are found to be excellent drug candidate based on the molecular docking studies and its regression studies.

Thus on the basis of regression analysis study and docking study of substituted pyrimidine derivatives, it can be concluded that these compounds on further studies may prove to be therapeutic agent against mycobacterium tuberculosis.

\section{References}

[1] L. H. Hall, "A Structure-Information Approach to the Prediction of Biological Activities and Properties," CHEMISTRY \& BIODIVERSITY , vol.1, p.183, 2004.

[2] P. N.Judson, "QSAR and Expert System in Prediction of," Pestic.Sci., pp. 155-160, 1992.

[3] A. Tropsha, "Best Practices for QSAR Model Development, Validation and Exploitation," Mol. Inf., pp. 476-488, 2010.

[4] L. H. L.B. Kier, "Quantitative Information Analysis: The New Center of Gravity in Medicinal Chemistry," Medicinal Chemistry Research, vol. 7, pp. 335-339, 1997.

[5] L. H. H. L. B. Kier, Molecular Structure Description: The Electrotopological State, Academic Press, 1999.

[6] L. B. K. L. H. Hall, Topological Indices and Related Descriptors in QSAR and QSPR, UK, 1999.

[7] J. A. B. George W. Adamson, "Evaluation of an empirical structure-activity relationship for property prediction in a structurally diverse group of local anaesthetics," Journal of the Chemical Society, Perkin Transactions 1, no. 2, pp. 168-172, 1976.

[8] Shams Uzzaman and Ayaz Mahmood Dar, "PATHWAYS FOR THE SYNTHESIS OF PYRIMIDINE AND PYRAN BASED HETEROCYCLIC DERIVATIVES:A CONCISE REVIEW," Eur. Chem. Bull., vol. 4, no. 5, pp. 249-259, 2015. 
[9] Vinita Sharma, et. al., "Significance and Biological Importance of Pyrimidine in the Microbial World," International Journal of Medicinal Chemistry, p. 31, 2014.

[10] V. H. Babu, P. S. Kumar, K. K. Srinivasan, and G. V. Bhat, "Synthesis, antitumor and antibacterial activities of certain substituted pyrimidines bearing benzofuran,” Indian Journal of Pharmaceutical Sciences, vol. 66, no. 5, pp. 647-652, 2004.

[11] Sander T1, Freyss J, von Korff M, Rufener C. "DataWarrior: an open-source program for chemistry aware data visualization and analysis" J Chem Inf Model., 55(2), 460-73, 2015.

[12] J. Huuskonen, "Estimation of Aqueous Solubility for a Diverse Set of Organic Compounds Based on Molecular Topology," Journal of Chemical Information and Modeling, pp. 773-777, 2000.

[13] J. M. B. W. Y. W. S. G. F. P. K. Annamaria Lilienkampf, "Structure-Activity Relationships for a Series of Quinoline-Based Compounds Active against," Journal of Medicinal Chemistry, vol. 52, p. 2109-2118, 2009.

[14] A. V. A. ,. N. K. P.,. I. H. C. Sumesh Eswaran, "Design and synthesis of some new quinoline-3-carbohydrazone derivatives as potential antimycobacterial agents," Bioorganic \& Medicinal Chemistry Letters, p. 1040-1044, 2010.

[15] P. M. R. a. B. G. K. Ganatra S. H., "Inhibition Studies of Pyridine Based Compounds on Quinolinic Acid Phosphoribosyltransferase Enzyme as A Potent Anti-Tuberculosis Agent" Asian J. Research Chem, vol. 5, no. 9, pp. 1159-1165, 2012.

[16] Kai-Cheng Hsu, Yen-Fu Chen, Shen-Rong Lin, Jinn-Moon Yang. "iGEMDOCK: a graphical environment of enhancing GEMDOCK using pharmacological interactions and post-screening analysis" BMC Bioinformatics 2011, 12(Suppl 1):S33

[17] P. N. Judson, "QSAR and Expert Systems in the Prediction of," Pestic. Sci., pp. 155-160, 1992.

[18] L. H. H. L. B. Kier, Research Studies Press, 1986.

[19] Sunita Patel Hardia, "Topological Modeling of log D7.4 of Hydroxylated Aromatic Aldehydes" IJSRCS, Vol.2 , Issue.1 , pp.1-4, Dec-2015.

[20] Asmita Sharma and Anubha Vijay Pandya, "Modeling of sulfonamide using NMR chemical shift by QSDAR Method", International Journal of Scientific Research in Chemical Sciences, Vol.1, Issue.1, pp.1-8, 2014.

[21] A. K. Parmar, M. R. Patle, "Regression Analysis and Docking Study of Hydroxyl Quinoline Based Compounds as Anti-Tuberculosis Therapeutic Agents", International Journal of Scientific Research in Biological Sciences, Vol.6, Issue.1, pp.177-186, 2019.

[22] SPSS version 24 for Windows. SPSS software packages, SPSS Inc., 444 North Michigan Avenue, Suite 3000, Chicago, Illinois, 60611, USA. 